\title{
Critical Wetting of a Liquid/Vapor Interface by Octane
}

\author{
T. Pfohl and H. Riegler \\ Max-Planck-Institut für Kolloid- und Grenzflächenforschung, Rudower Chaussee 5, D-12489 Berlin, Germany
}

(Received 3 April 1998; revised manuscript received 29 July 1998)

\begin{abstract}
The wetting of the planar interface between air saturated with octane vapor and an aqueous solution by octane is investigated by ellipsometry and interfacial tension measurements. The interfacial interactions are varied through the concentration of the uncharged solute (glucose). We observe abrupt and continuous increases of the octane coverage with growing solute concentration. This we attribute to short- and long-range (dispersion) interactions, respectively. The continuous thickness growth is analyzed as a critical wetting transition. [S0031-9007(98)08325-2]
\end{abstract}

PACS numbers: 68.10.-m, 64.70.-p, 68.45.Gd

Wetting is determined by interfacial forces. These are commonly divided into short-range components, whose origins are barely understood, and long-range components, which are generally attributed to fairly well understood dispersion forces [1]. Alkanes are nonpolar and electrically neutral. Therefore their dispersion force component is presumably important. Accordingly, the wetting of the air/water interface by alkanes has been the subject of several studies [2]. Recently, with such a system, a continuous wetting transition has been induced by changing the long-range dispersion forces via temperature variation [3]. Stepwise transitions due to modifications of the short-range interactions were triggered by variation of the sodium chloride subphase concentration [4].

In this Letter, we present a study on the wetting of an air/aqueous glucose solution interface by octane. We observe stepwise and continuous increases of the octane coverage with growing subphase solute concentration which we attribute to changes of short- and long-range interaction components, respectively. The continuous thickness growth at high glucose concentrations is identified as a critical wetting transition and analyzed quantitatively in some detail.

The octane $\left(\mathrm{C}_{8}\right.$, purity $>99 \%$, Sigma $)$ was purified chromatographically. A Teflon dish $(\phi \approx 30 \mathrm{~mm})$ filled with a solution of glucose (purity $99.5+$, Fluka, checked for surface active components) was kept in a gas-tight, thermostated $\left( \pm 0.1^{\circ} \mathrm{C}\right)$ compartment. Into this cell, next to the Teflon dish, we placed a drop of liquid bulk octane with sufficient volume for vapor saturation. The octane surface coverage was measured by ellipsometry (Beaglehole Instruments). For details on the measurement setup and the data analysis, see Pfohl et al. [5]. For thin films, the ellipticity is sensitive to the average layer thickness, the refractive index, its anisotropy, and the refractive index profile normal to the interface. Anisotropy effects are usually small [6], and details about the refractive index profile are unknown. Therefore a homogeneous refractive index is assumed (e.g., the bulk value), and a corresponding thickness is calculated which is a reasonable measure for the adsorbate coverage. The interfacial tensions were measured with the pendant drop and drop vol- ume method [7]. The refractive index of the glucose solution, $n$, is a linear function of the glucose concentration $c$ with $n=1.3326+0.0254 c(\lambda=589 \mathrm{~nm}, c$ in $\mathrm{mol} / \mathrm{l}$, $T=25^{\circ} \mathrm{C}$ ) as determined experimentally.

In control experiments, the ellipticities of the interface between the glucose solutions and octane-free air saturated with water vapor were measured. They are quite small and increase from $\approx 4 \times 10^{-4}$ for pure water to $\approx 7 \times 10^{-4}$ for concentrated glucose solutions $(3 \mathrm{~mol} / \mathrm{l})$. The ellipticity of the pure water surface is commonly attributed to its surface roughness of $\approx 2.5 \AA$ (see Beaglehole [5]). This roughness will barely be affected by the glucose because the surface tension between solution and air, $\gamma_{s / a}$, is increasing only from $\approx 72 \mathrm{mN} / \mathrm{m}$ to $\approx 76 \mathrm{mN} / \mathrm{m}$ (see Fig. 2). Hence, the observed ellipticity increase of $\approx 3 \times 10^{-4}$ is presumably due to a glucose depletion layer in agreement with fundamental thermodynamic interpretations of the slight increase of the surface tension. If this layer were a slab of pure water, its thickness would be $\approx 3 \AA$ according to the ellipsometry data. Hypothesized glucose concentration profiles other than a simple slab yield comparable thicknesses. Altogether, changes of the ellipticity due to interfacial glucose depletion are much smaller than contributions from the octane adsorption. Henceforth, they are neglected in the analysis of the ellipsometric octane adsorption data.

Figure 1 shows the equilibrium thicknesses of octane layers, $D$, adsorbed at the interface between air saturated with octane vapor and aqueous glucose solution as a function of the refractive index of the solution. The data are calculated with the refractive index of liquid bulk octane $\left[n\left(\mathrm{C}_{8}\right)=1.395(\lambda=589 \mathrm{~nm}), T=25^{\circ} \mathrm{C}\right]$. On pure water, there is only a submonolayer octane coverage. Already a small glucose concentration leads to "microscopic" ( $\approx 30 \AA)$, a slightly higher concentration (at $n \approx 1.335$ ) to "mesoscopic" ( $\approx 70 \AA)$, layer thicknesses (see inset in Fig. 1). Between $n \approx 1.335$ and $n \approx$ 1.370 , the thicknesses remain approximately unchanged. Then, between $n \approx 1.370$ and $n \approx 1.390$, a presumably continuous layer growth up to a thickness of $\approx 250 \AA$ is observed. No data are shown between $n=1.390$ and $n=1.400$ because the similarity of the film and subphase 


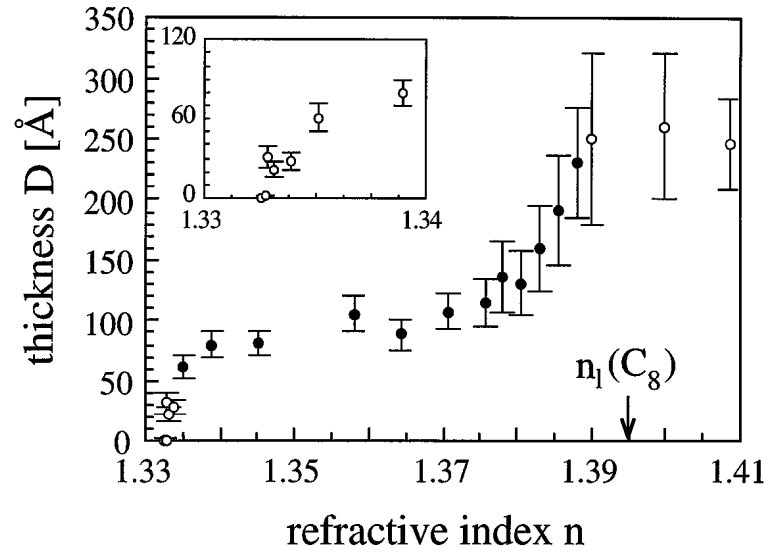

FIG. 1. Octane film thicknesses $D$ adsorbed at interfaces between air saturated with octane vapor and aqueous glucose solutions of various refractive indices $n$. The inset shows the data for low glucose concentrations in detail. The thicknesses are derived from ellipsometric data assuming homogeneous octane films with refractive indices of liquid octane $\left[n\left(C_{8}\right)=\right.$ 1.395]. Data points not used in the fit of Fig. 4 (below) are marked by open circles.

refractive indices allows no credible thickness determination. For $n>1.400$, the thickness remains at $\approx 250 \AA$.

Figure 2 shows various interfacial tensions: (1) $\gamma_{s / a}$, the interfacial tensions of glucose solution ("s") versus air (" $a$ "), (2) $\gamma_{s / o}$, the interfacial tensions of glucose solution versus bulk octane ("o"), and (3) $\gamma_{s / a}^{i}$, the initial spreading coefficients calculated from $\gamma_{s / a}^{i}=\gamma_{s / a}-$ $\left(\gamma_{s / o}+\gamma_{o / a}\right) \cdot \gamma_{s / a}^{i}$ increases approximately linearly with the glucose concentration. For pure water, it is slightly negative [8]. At $n \approx 1.34$, it changes sign and reaches $\approx+5 \mathrm{mN} / \mathrm{m}$ at $n=1.41$. According to definition, the initial spreading coefficient represents the interfacial inter-

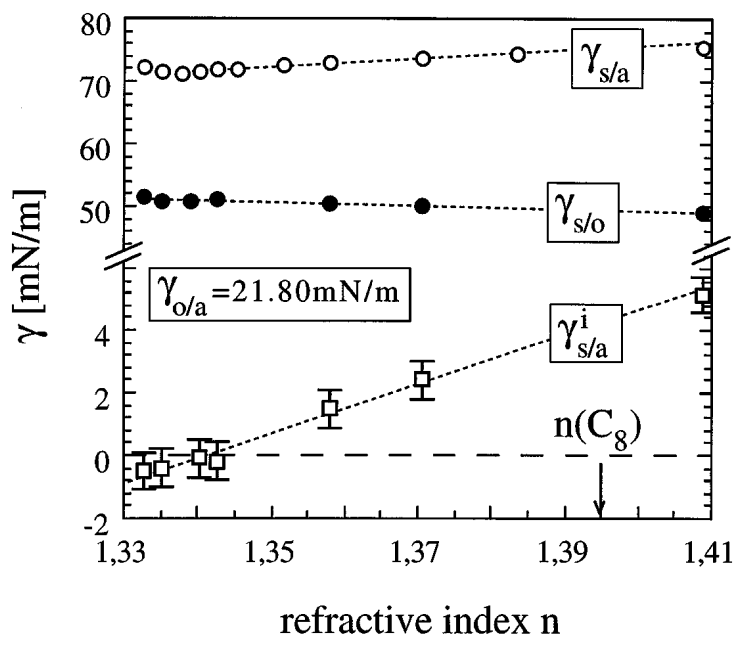

FIG. 2. Interfacial tensions between air saturated with octane vapor and aqueous glucose solutions $\left(\gamma_{s / a}\right)$, between the solutions and bulk octane $\left(\gamma_{s / o}\right)$, and the derived initial spreading coefficient $\left(\gamma_{s / a}^{i}\right)$, as a function of the refractive index of the solutions, $n$. action component when long-range dispersion forces are neglected [9]. For $\gamma_{s / a}^{i}<0$, this component disfavors initial spreading, whereas $\gamma_{s / a}^{i}>0$ prompts initial spreading (not necessarily complete wetting).

The comparison of the data of Figs. 1 and 2 reveals a remarkable feature. The increasing subphase refractive index is accompanied by an approximately linear increase of the interfacial energies including $\gamma_{s / a}^{i}$. This is contrasted by the clearly nonlinear growth of the octane layer thickness. This indicates different growth laws of wetting at low and high glucose concentrations, respectively. The growth from submonolayer to microscopic and mesoscopic thicknesses occurs in a refractive index range where $\gamma_{s / a}^{i}$ changes its sign. Hence, these transitions are supposedly caused by changes of the short-range interaction components. The origin of short-range interactions is theoretically not well understood. Therefore these transitions will not be analyzed here any further. In the range of the continuous layer thickness growth at $n>1.36, \gamma_{s / a}^{i}$ is definitely positive without any characteristic behavior (kinks, etc.). This growth regime is therefore attributed to changes of the long-range, i.e., dispersion force interactions.

Theory shows that if the interfacial interaction potential $V(D)$ consists of different short- and long-range components, transitions between films of microscopic and mesoscopic thicknesses [10] as well as critical wetting, i.e., a continuous wetting transition to macroscopic thicknesses [1], may occur. Theoretical descriptions of critical wetting [1] develop the potential $V(D)$ around the transition in a power series:

$$
V(D)=\frac{c_{1}}{D^{2}}+\frac{c_{2}}{D^{3}}+\ldots
$$

Equation (1) describes critical wetting if $c_{1}$ is negative below the critical parameter value and positive above. $c_{2}$ has to be positive throughout. For dispersion forces, $c_{1}$ is proportional to the Hamaker constant which is linked to the subphase refractive index $n$ [11]. Thus, in the vicinity of the wetting transition, one can approximate (with $n_{w}$ as a critical refractive index):

$$
c_{1} \propto\left(n-n_{w}\right) / n_{w} .
$$

For $c_{1}$ approaching zero, Eqs. (1) and (2) yield a critical exponent of $-1[1,3]$ :

$$
D \propto\left|\frac{n-n_{w}}{n_{w}}\right|^{-1} .
$$

Figure 3 depicts interfacial potentials in the regime of the critical wetting transition as described by Eq. (1) for various subphase refractive indices, consistent with the observed continuous octane layer thickness growth. Short(dotted lines) and long-range (dashed lines) components contribute to the combined potential (solid lines). Below the transition [Figs. 3(a) and 3(b)] the short-range component favors wetting, whereas the long-range contribution is nonwetting. Thus the combined potential has a minimum 


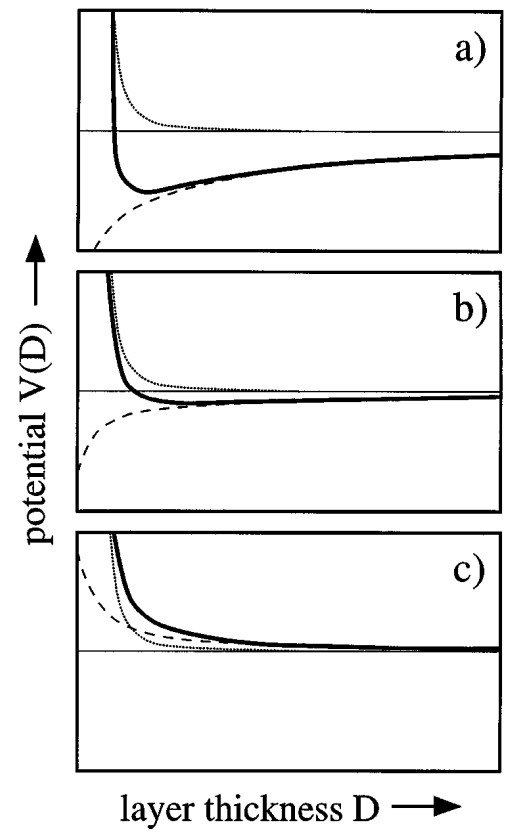

FIG. 3. Schematic of the interfacial potential $V(D)$ (solid lines) consisting of short- (dotted lines) and long-range (dashed lines) components according to Eq. (1) in the vicinity of the critical wetting transition: (a) Short-range components wetting and long-range components nonwetting, respectively, causing a potential minimum; (b) similar to (a) but with a decreased strength of the nonwetting long-range component causing a shift of the potential minimum to larger $D$; (c) both components favoring wetting.

corresponding to a certain equilibrium film thickness. With the long-range component becoming continuously less nonwetting, due to a decrease of $c_{1}$ according to Eq. (2), the minimum of the combined potential is continuously shifted to thicker films [Fig. 3(b)]. This agrees with the experimentally observed continuous thickness increase between $n=1.360$ and $n=1.390$. Macroscopic film thicknesses are expected for $c_{1}>0$, when both force components favor wetting [Fig. 3(c)]. In the real experiment, only limited thicknesses of $\approx 250 \AA$ are observed in this regime. This discrepancy is vindicated later.

The Hamaker constants $A_{s / o / a}$ calculated [12] for the system solution/octane/air are positive for low glucose concentrations. They become negative at $n=1.388$ thus favoring wetting at high glucose concentrations.

Figure 4 demonstrates that the experimental data can well be fitted to a critical wetting transition at $n_{w} \approx 1.390$ in agreement with the independently estimated Hamaker constant and the theoretically predicted critical exponent of -1 . The fit is based on data from $n>1.335$ to $n<1.390$ only. The excluded data are either from the range with $c_{2}<0$ at low $n$ or presumably very close to or in the complete wetting regime at high $n$ where the experimental conditions limit the layer thicknesses. For a correct data analysis according to critical wetting, it is further necessary to fit only the dispersion force related, continuous layer

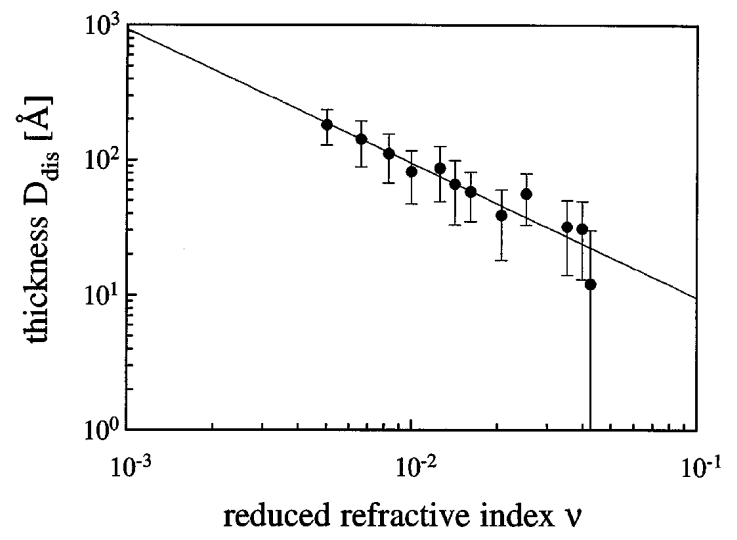

FIG. 4. Octane coverage $D_{\text {dis }}$ attributed to long-range dispersion forces $\left(D_{\text {dis }}=D-D_{\text {sr }}\right)$ as a function of the reduced refractive index $\nu$ of the glucose solution $\left[\nu=\left(n_{w} / n\right)-1\right]$. The solid line is a fit with an offset film thickness $D_{\text {sr }}=48 \AA$.

coverage increase (" $D_{\text {dis }}$ ") to Eq. (1) and subtract a shortrange induced "offset" layer thickness (" $D_{\text {sr }}$ "):

$$
D_{\text {dis }}=D-D_{\text {sr }} \text {. }
$$

Thus, with a critical exponent, $x$, of -1 , a fit of the experimental data with Eqs. (1) and (4) yields $D_{\text {sr }}=$ $48 \pm 12 \AA$ and $n_{w}=1.394 \pm 0.005$. These numbers agree well with the experimentally evident $D_{\text {sr }}$ and the independently calculated $n_{w}$. Nevertheless, it should be remarked that the experimental scatter also allows for other fits within the error margins (e.g., $n_{w}=1.400, x=-1.7$, $D_{\text {sr }}=59 \pm 15 \AA$ ).

The data can also be analyzed by assuming an alkane layering profile with a densely packed molecularly thin alkane slab at the interface (Fig. 5). Theory [13] and experiment [14] indicate that alkanes close to the interface are more densely packed than in the distant bulk (with a refractive index increased by $\Delta n_{\text {sr }}$ ). Thus the interfacial dispersion forces are described by a disjoining pressure $\Pi$ $[11,15]$ :

$$
\Pi(D)=\frac{A_{134}-A_{132}}{6 \pi\left(D_{\mathrm{dis}}+D_{\mathrm{sr}}^{0}\right)^{3}}-\frac{A_{134}}{6 \pi D_{\mathrm{dis}}^{3}} .
$$

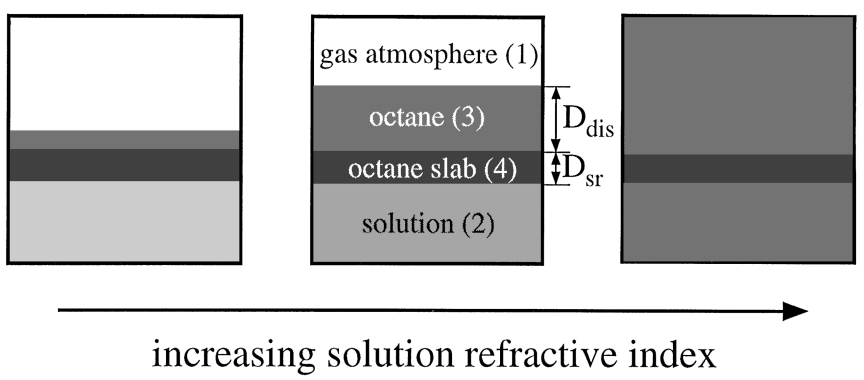

FIG. 5. Schematic of the layering model with an interfacial octane slab of constant thickness $D_{\text {sr }}$ and increased density. $D_{\text {dis }}$ is the octane coverage attributed to dispersion forces. 
This describes a potential with a minimum [16] and thus a finite film thickness if $A_{134}<0$ (denser interfacial alkane slab) and $A_{132}>0$. One obtains an equilibrium film thickness $D_{\text {dis }}\left(\alpha=-A_{134} / A_{132}\right)$ :

$$
D_{\mathrm{dis}}=\alpha D_{\mathrm{sr}}^{0}\left[1+\left(1+\frac{1}{\alpha}\right)^{2 / 3}+\left(1+\frac{1}{\alpha}\right)^{1 / 3}\right] \text {. }
$$

$D_{\text {dis }}$ grows proportional to $\left|A_{132}\right|^{-1}$ if $A_{132} \rightarrow 0$; i.e., with the same critical exponent as in the approach according to Eq. (1). To a first approximation $A_{132} \propto n\left(\mathrm{C}_{8}\right)-$ $n_{s}\left[n_{s}=n\right.$ (solution) $]$ and $A_{134} \propto \Delta n_{\mathrm{sr}}=n_{\mathrm{sr}}-n\left(\mathrm{C}_{8}\right)$ [ $n_{\mathrm{sr}}=n$ (octane slab), $\Delta n_{\mathrm{sr}}$ assumed constant]. A fit of the data yields $D_{\mathrm{sr}}^{0}=25 \pm 9 \AA$ and a density (derived from $\left.n_{\text {sr }}\right)$ of $\rho(\mathrm{slab}) \approx 105 \% \times \rho\left(\mathrm{C}_{8}\right)$. The fitted curve is virtually identical to that of Fig. 4 (even when the increased alkane density at the interface is incorporated in the ellipsometric data analysis).

Experimentally, the layer thicknesses do not diverge at and above the transition at $n \approx 1.390$, contrary to theoretical predictions. This is typical for adsorption studies under complete wetting conditions [3,4] and attributed to incomplete saturation due to minor temperature gradients in the measurement cell. Retardation effects only increase the exponents of the algebraic decay of the dispersion force which has no influence on the curvature of the combined potential in principle.

In conclusion, we observe abrupt and continuous increases of the octane coverage at the interface between air saturated with octane vapor and an aqueous solution for low and high glucose concentrations, respectively. Interfacial tension measurements indicate that the adsorption steps at low glucose concentrations are due to changes of short-range interactions. The continuous thickness growth at high glucose concentrations is interpreted as a critical wetting transition induced by changes of long-range dispersion forces. Calculations confirm that the Hamaker constant changes sign close to this transition. For a data analysis in line with conventional critical wetting theory, an "offset" octane film of mesoscopic thickness caused by short-range interactions is taken into account. We alternatively propose a layering model based on an interfacial octane slab of increased density. The critical exponents, offset thicknesses, and transition points derived from the experimental data agree well with theoretical predictions of both models and corroborate the critical wetting interpretation.

We thank Helmuth Möhwald, Joseph Indekeu, Mark Auch, Britta Beyer, and Ingeborg Bartsch for their support.

[1] M. Schick, in Liquids at Interfaces, Proceedings of the Les Houches Summer School, edited by J. Charvolin et al. (North-Holland, Amsterdam, 1990), p. 415;
S. Dietrich, in Phase Transitions and Critical Phenomena, edited by C. Domb and J. Lebowitz (Academic, London, 1988), Vol. 12, p. 1; P. G. de Gennes, Rev. Mod. Phys. 57, 827 (1985).

[2] F. Hauxwell and R.H. Ottewill, J. Colloid Interface Sci. 34, 473 (1970); C. Del Cerro and G. J. Jameson, J. Colloid Interface Sci. 78, 362 (1980); F. Hauxwell, Langmuir 8, 602 (1992); B. A. Pethica, Langmuir 12, 5851 (1996); A. Lou and B.A. Pethica, Langmuir 13, 4933 (1997); D. Baumer and G. H. Findenegg, J. Colloid Interface Sci. 85, 118 (1982); K. Ragil et al., J. Chem. Phys. 105, 5160 (1996).

[3] K. Ragil et al., Phys. Rev. Lett. 77, 1532 (1996).

[4] N. Shahidzadeh et al., Phys. Rev. Lett. 80, 3992 (1998); A. Dussaud and M. Vignes-Adler, Langmuir 13, 581 (1997).

[5] T. Pfohl et al., Langmuir 14, 5285 (1998); P. Drude, Ann. Phys. Chem. 43, 126 (1891); D. Beaglehole, Physica (Amsterdam) 100B, 163 (1980); D. Beaglehole, J. Phys. (Paris), Colloq. 44, C10-147 (1983); J. Lekner, Theory of Reflection (Martinus Nijhoff Publishers, Dordrecht, 1987).

[6] M. Paudler et al., Langmuir 8, 184 (1992).

[7] A. Semmler et al., Langmuir 12, 4165 (1996); F.C. Goodrich, in Surface and Colloid Science, edited by E. Matijevic (Wiley-Interscience, New York, 1969), Vol. 1.

[8] T. Takii and Y.H. Mori, J. Colloid Interface Sci. 161, 31 (1993); S. Akatsuka et al., J. Colloid Interface Sci. 172, 335 (1995).

[9] F. Brochart-Wyart and J. Daillant, Can. J. Phys. 68, 1084 (1990); F. Brochart-Wyart et al., Langmuir 7, 335 (1991); G. J. Hirasaki, in Contact Angle, Wettability and Adhesion, edited by K.L. Mittal (VSP, Utrecht, The Netherlands, 1993), p. 183.

[10] For a detailed computation of $V(D)$ in Cahn theory including long-range forces, see J.O. Indekeu et al., J. Stat. Phys. (to be published).

[11] J. Israelachvili, Intermolecular \& Surface Forces (Academic Press, London, 1991).

[12] The approach of [11] was used because it predicts that pentane and longer alkanes do not completely wet water at room temperature in agreement with experiments and more sophisticated calculations [D. B. Hough and L. R. White, Adv. Colloid Interface Sci. 14, 3 (1980)], contrary to other approaches [P. Richmond et al., J. Colloid Interface Sci. 45, 69 (1973)]. For the calculation of $A_{s / o / a}$ the small zero-frequency contribution is assumed independent from the glucose concentration.

[13] D. Y. Yoon et al., in Monte Carlo and Molecular Dynamics Simulations in Polymer Science, edited by K. Binder (Oxford University Press, New York, 1995), p. 433.

[14] The ellipticities of interfaces between bulk alkane and silicon wafers indicate an increased alkane density at the interface (T. Pfohl, Ph.D. dissertation, University of Potsdam, Germany, 1998).

[15] M.P. Valignat et al., Langmuir 9, 601 (1993); 9, 3255 (1993).

[16] I. M. Tidswell et al., Phys. Rev. B 44, 10869 (1991); V. B. Shennoy and W. F. Saam, Phys. Rev. Lett. 75, 4086 (1995). 